\title{
Numerical Simulation of Microstructure of Workpiece in Closed-open Cross Wedge Rolling
}

\author{
Zi-rong ZHOU ${ }^{1,}$, Xue-dao $\mathrm{SHU}^{1, \mathrm{~b}}$, Ji-dong MA ${ }^{1, \mathrm{c}}$, Chang $\mathrm{SHU}{ }^{2, \mathrm{~d}}$ \\ ${ }^{1}$ Faculty of Mechanical Engineering and Mechanics, Ningbo University, Ningbo, 315211, China \\ ${ }^{1}$ Zhejiang Provincial Key Laboratory of Part Rolling Technology, Ningbo, 315211, China \\ ${ }^{2}$ School of Mechanical and Automotive Engineering, Hefei Polytechnic University, Hefei, 230009, \\ China \\ a1165420916@qq.com, bshuxuedao@nbu.edu.cn, 'c328885877@qq.com, dshuchang950107@163. \\ com
}

Keywords: Closed-open Cross wedge rolling, Microstructure, FEM, Numerical simulation

\begin{abstract}
Closed-open cross wedge rolling(CWR) is a kind of advanced precision forming technology, which can effectively reduce or even eliminate the concave. The workpiece is processed from the shaft end to the middle by the rolls first, and then machined by open CWR. An auto pump shaft is taken as the research object. A 3D coupling thermal, mechanical, microstructural finite element model is established by the finite element analysis software DEFORM-3D according to the constitutive equation and microstructure evolvement model of 42CrMo. Distribution laws of temperature field and microstructure field are acquired. This work not only lays a theoretical foundation for improving the product quality and the comprehensive performance of rolled piece, but also provides a guideline for further application and extension of closed-open CWR.
\end{abstract}

\section{Introduction}

The quality of CWR product is not just evaluated by the basic shape, but also the comprehensive mechanical properties of rolled pieces due to the continuous development of CWR technology. In order to reflect the advantages of CWR plastic forming technology, it is necessary to ensure that the product can not only meet the basic requirements of shape and size, but also the comprehensive performance. Further research is warranted to study the microstructure evolution during CWR process because mechanical properties of products are the comprehensive performance of microstructure.

Z.H. Hu etal ${ }^{[1-2]}$ from the University of Technology and Science Beijing derive change rules of stress field, strain field, temperature field and micro grain size by numerical simulation and rolling experiment. N.Bontcheva and G.Petzov ${ }^{[3-4]}$ from Bulgarian Academy of Sciences couple the recrystallization behavior and the grain growth process of the metal during the forming process. The recrystallization dynamic model and history data of austenite grain growth process are obtained. Y.C. Lin etal ${ }^{[5-6]}$ from Central South University derive the constitutive equations of rheological stress of 42CrMo steel under high temperature condition based on the thermal simulation experiment and metallographic experiment, and then study the microstructure evolution law of 42CrMo steel during high temperature deformation. M.T. Wang etal $^{[7]}$ from Yanshan University investigate the microstructure evolution of workpiece during single-wedge rolling process according to the typical process parameters of CWR. The distribution temperature field, strain field and austenite grain size are gained.

In summary, the present research about microstructure of CWR workpiece focuses in single or multi wedge rolling which belongs to open CWR. The research on the microstructure of closed-open CWR workpiece is still a gap. The automobile oil pump shaft (as shown in Fig.1) is taken as the research object. A 3D coupling thermal, mechanical, microstructural finite element model is developed by PROE and DEFORM according to the constitutive equation and dynamic recrystallization model of $42 \mathrm{CrMo}$. The distribution and variation of rolled piece during closed-open CWR are studied. 


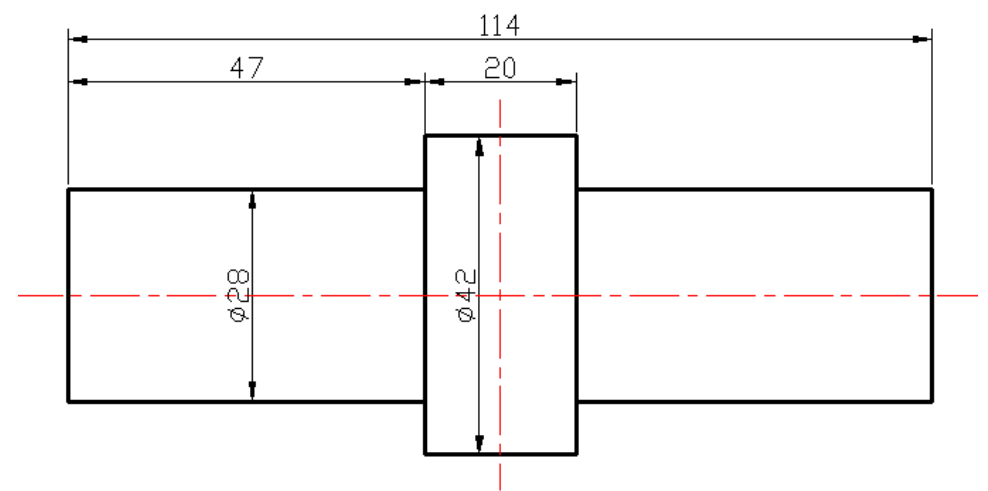

Fig. 1. Dimensions of the workpiece

\section{Finite Element Modeling of Closed-open CWR}

Constitutive Equation and Microstructure Evolvement Model of 42CrMo

Real stress-strain curve of 42CrMo is gained by thermal analog test and on this basis constitutive equations of rheological stress is built. Microstructure evolvement model of 42CrMo is obtained based on metallographic experiment. According to references ${ }^{[5-6]}$, we have the following equations.

Constitutive equation:

$$
\dot{\varepsilon}=1.34 \times 10^{18}\left[\sinh \left(8.198 \times 10^{-3} \sigma\right)\right]^{8.1434} \times \exp \left(-\frac{4.6334 \times 10^{5}}{R T}\right)
$$

Dynamic recrystallization equations:

$$
\left\{\begin{array}{l}
\varepsilon_{p}=7.28 \times 10^{-4} d_{0}^{0.31}\left[\dot{\varepsilon} \exp \frac{247000}{R T}\right]^{0.21} \\
\varepsilon_{c}=0.7 \varepsilon_{p} \\
\varepsilon_{0.5}=3.0 \times 10^{-3} d_{0}^{0.4} \dot{\varepsilon}^{0.086} \exp \left(\frac{4089}{T}\right) \\
X_{\text {drex }}=1-\exp \left[-0.693\left(\frac{\varepsilon-\varepsilon_{c}}{\varepsilon_{0.5}-\varepsilon_{c}}\right)^{1.8}\right] \\
d_{d r e x}=38 d_{0}^{0.8} \dot{\varepsilon}^{-0.234} \exp \left(-\frac{42327.5}{R T}\right)
\end{array}\right.
$$

Grain growth model:

$$
d=\left(d_{0}^{3.55}+2.90 \times 10^{25} \exp \left(\frac{-64273.96}{T}\right) \cdot t\right)^{\frac{1}{3.55}}
$$

Where $\varepsilon_{p}$ is peak strain of dynamic recrystallization; $\varepsilon_{c}$ is critical strain of dynamic recrystallization; $T$ is deformation temperature; $X_{\text {drex }}$ is effect coefficient of dynamic recrystallization; $\varepsilon_{0.5}$ is Strain in dynamic recrystallization volume fraction of $50 \%$; $d_{d r e x}$ is grain size of dynamic recrystallization.

Rolling Model

The definitions of the model are:(1) Half of the workpiece geometry is used and a symmetry constraint is applied on the symmetry plane. (2) The rolls are defined as rigid bodies while workpiece is defined as a formable part. (3) The friction between the roller and rolling is shear friction, and the friction coefficient is set to 2. (4) The rolling temperature is set to $1050^{\circ} \mathrm{C}$ and the roller speed is set to $10 \mathrm{r} / \mathrm{min}$. The process parameters of the dies and workpiece are shown in Tab. 1. 
Tab.1

The Process Parameters of closed-open CWR

\begin{tabular}{ccccc}
\hline $\begin{array}{c}\text { Forming angle } \\
\text { of closed CWR } \\
\alpha_{1} /\left(^{\circ}\right)\end{array}$ & $\begin{array}{c}\text { Spreading angle } \\
\text { of closed CWR } \\
\beta_{1} /\left(^{\circ}\right)\end{array}$ & $\begin{array}{c}\text { Forming angle } \\
\text { of open CWR } \\
\alpha_{2} /\left(^{\circ}\right)\end{array}$ & $\begin{array}{c}\text { Spreading angle } \\
\text { of open CWR } \\
\beta_{2} /\left(^{\circ}\right)\end{array}$ & Reduction $\psi /(\%)$ \\
\hline 29 & 8 & 32 & 10 & 55.6 \\
\hline
\end{tabular}

Import constitutive equation and microstructure evolvement model of 42CrMo and set heat exchange surface. The heat exchange coefficient is set to $11 \mathrm{~W} /\left(\mathrm{m}^{2} \cdot \mathrm{K}\right)$, and the convective heat transfer coefficient of air is set to $0.02 \mathrm{~W} /\left(\mathrm{m}^{2} \cdot \mathrm{K}\right)$. The initial grain size is set to $150 \mathrm{um}$ according to the experimental value. Finite element model is finally established as shown in Fig. 2.

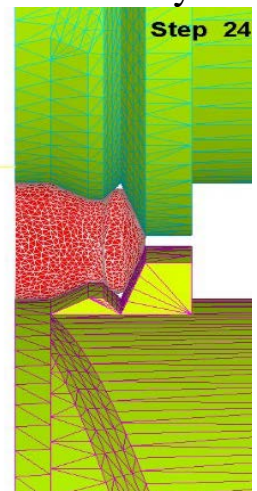

Fig. 2. Finite element model

\section{Distribution of Temperature Field During Closed-open CWR}

Fig. 3 shows the temperature distribution of workpiece in the longitudinal section during the rolling process. As can be seen from this figure, the temperature of the contact region between the workpiece and forming wedge drops in the wedge stage and spreading stage of closed CWR. There is no significant change in the metal temperature of the core because the deformation is not penetrated into the core, and the heat dissipation condition is relatively poor.

During the finishing stage of the closed CWR, the loss of heat is greater than the thermal energy from the plastic work transformation, which leads to a decrease in the temperature of the contact area. The temperature of the core metal at the axial end rises. This is because the hindering effect of the block wedge makes the metal at the axial end flow in the radial direction, resulting in a large plastic deformation which generates a large quantity of heat.

At the end, the temperature of shaft end drops below the rolling temperature because almost no plastic work is transformed into heat, but the temperature is still higher than the other parts due to the deformation caused by the block wedge and poor heat dissipation conditions of the middle part. 

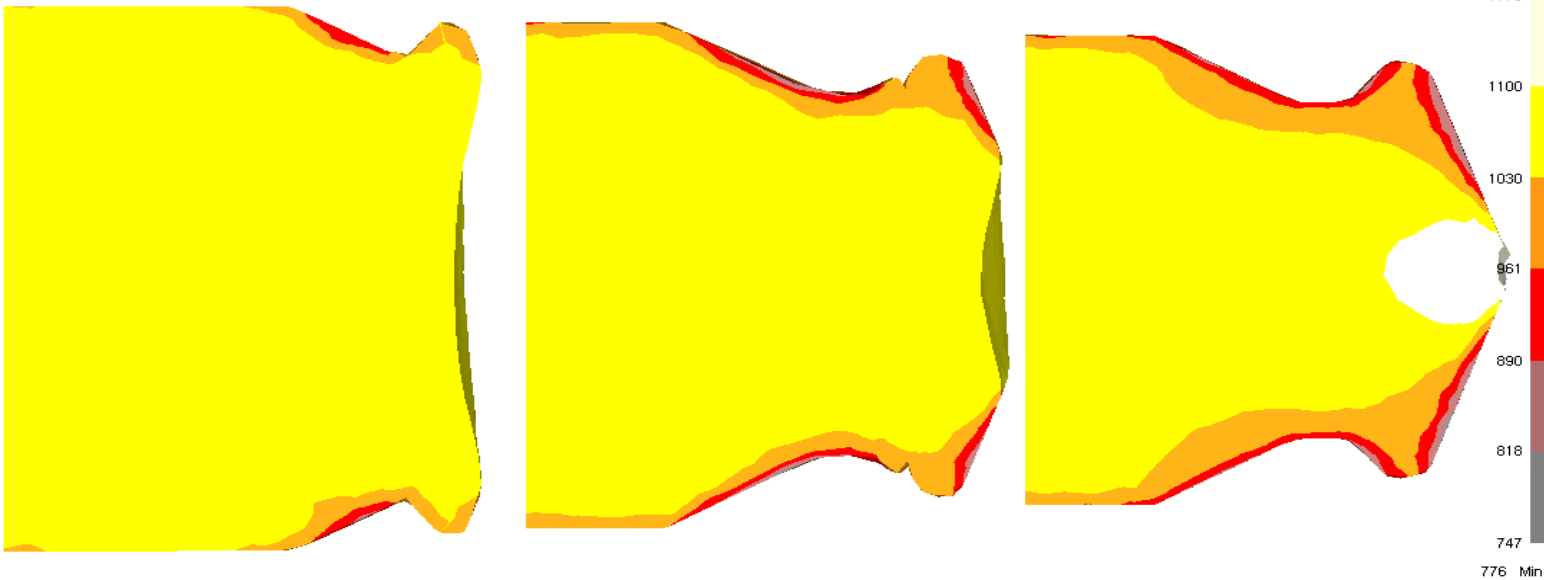

(a) Wedging stage of closed CWR

(b) Spreading stage of closed CWR

(c) Finishing stage of closed CWR
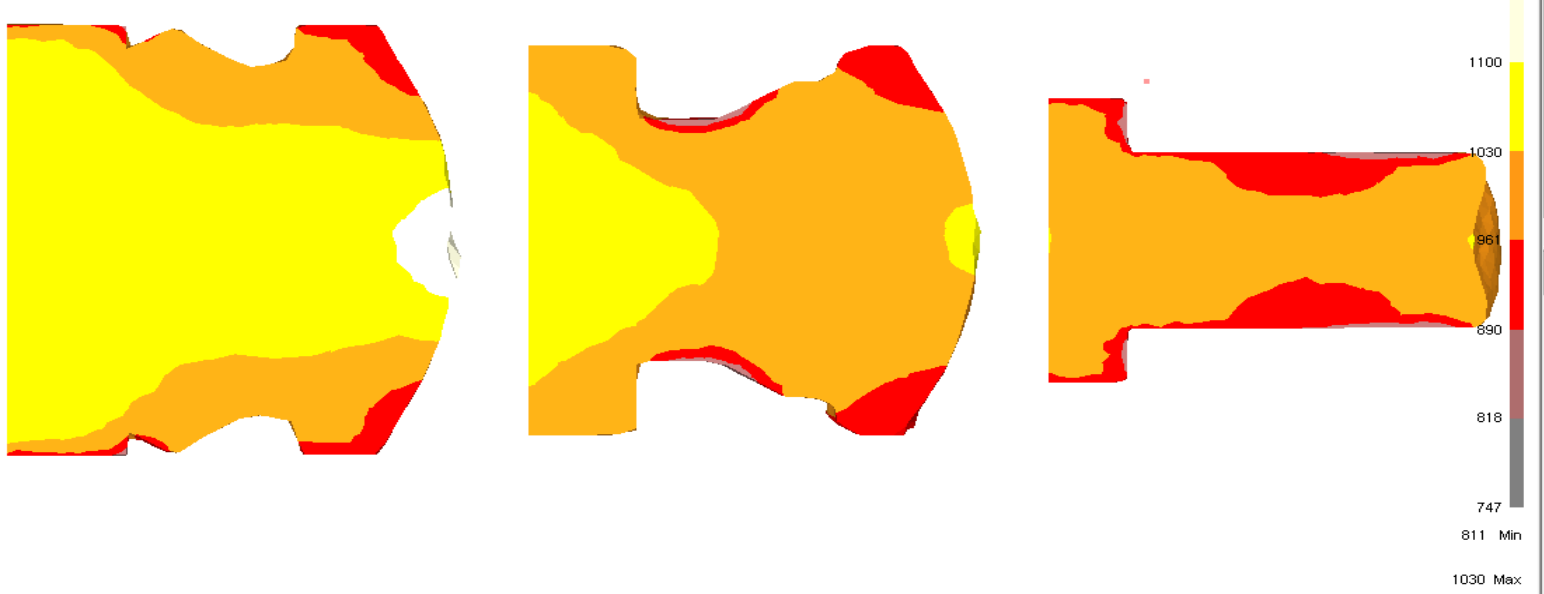

(d) Wedging stage of open CWR

(e) Spreading stage of open CWR

(f) End of rolling

Fig. 3. Temperature distribution of workpiece in the longitudinal section during closed-open CWR process.

\section{Distribution of Microstructure Field During Closed-open CWR}

Fig.4-Fig.8 show the distribution of microstructure field during closed-open CWR. According to the volume fraction of dynamic recrystallization of the longitudinal section of the workpiece, high temperature and large deformation are more likely to occur dynamic recrystallization. High strain rate which is not conducive to recrystallization will increase the critical strain value of dynamic recrystallization. But the large deformation caused by the repeated rolling by closed-open CWR is sufficient to offset the effect of high strain rate, which leads to the dynamic recrystallization and the grain size decrease obviously, and thus it can be a driving force for dynamic recrystallization in the deformed region and obvious decrease in grain size. There is almost no recrystallization and no significant changes in grain size near the core of them metal as a result of not more than the critical deformation of dynamic recrystallization. Metal in the forming zone reached complete recrystallization The volume fraction of dynamic recrystallization is only large on the rolling workpiece surface, and it is few in the rest part of the workpiece during the spreading stage of open CWR. This results from the fact that smaller deformation cannot penetrate deep into the center of workpiece. 


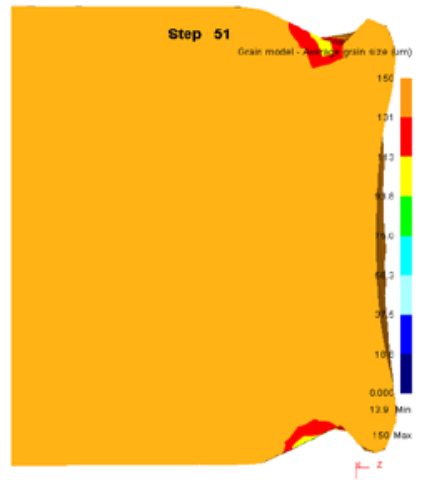

(a) Average grain size

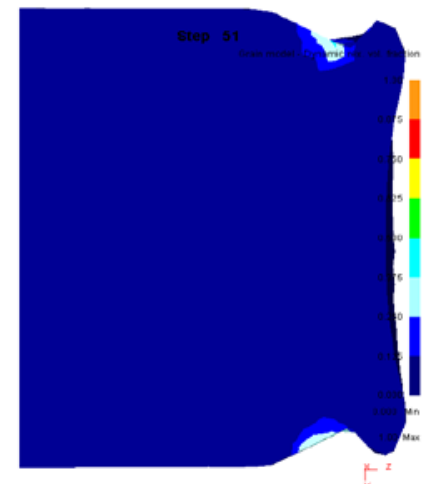

(b) volume fraction

Fig. 4. Average grain size and volume fraction of closed wedging zone

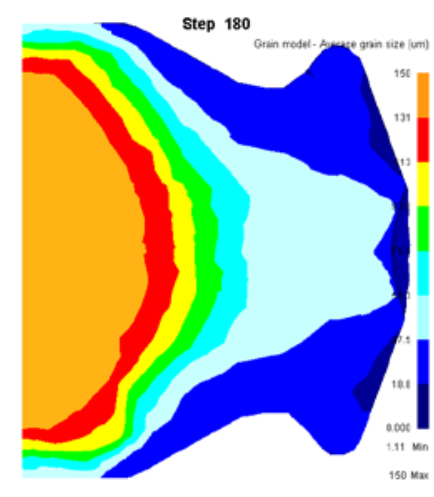

(a) Average grain size

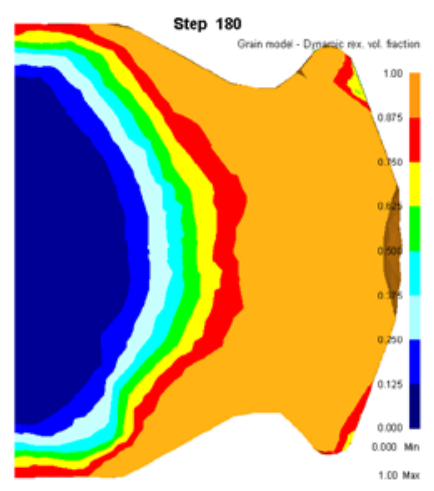

$t^{2}=$

Fig. 5. Average grain size and volume fraction of closed spreading zone

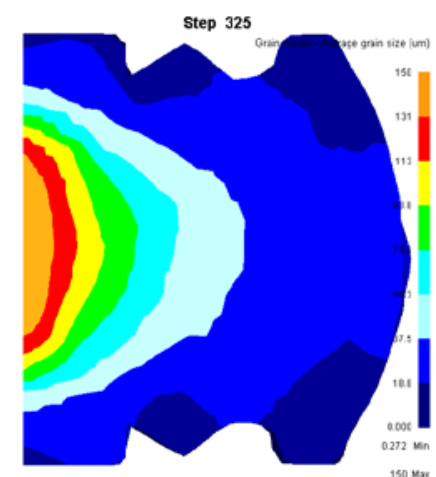

(a) Average grain size

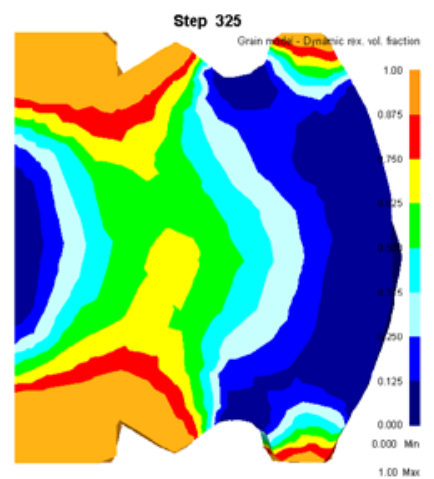

(b) volume fraction

Fig. 6. Average grain size and volume fraction of open wedging zone 


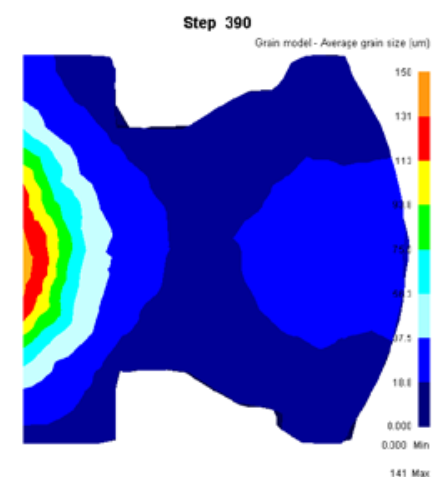

(a) Average grain size

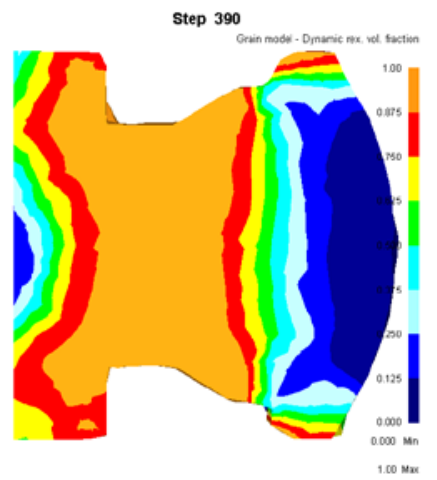

(b) volume fraction

Fig. 7. Average grain size and volume fraction of open spreading zone

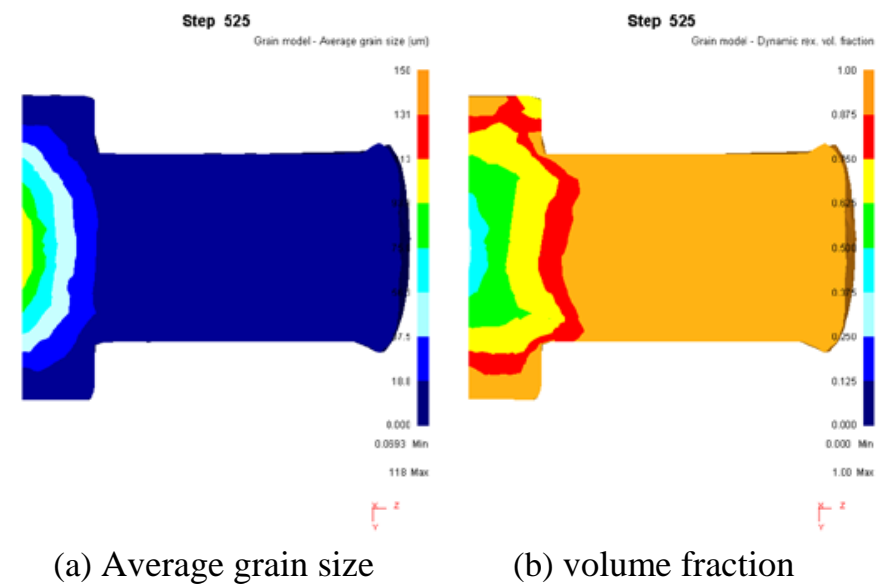

Fig. 8. Average grain size and volume fraction of open finishing zone

\section{Summary}

1) The increased heat by plastic work and the lost heat by thermal contact are essentially flat and there is a slight drop in the temperature of the contact part between workpiece and roller in closed wedging stage and open wedging stage. The heat generated from plastic deformation is far greater than energy loss by heat transfer and radiation, resulting in the sharp rise of the rolling temperature.

2) The dislocation accumulation of rolling deformation stage provides adequate storage for dynamic recrystallization. Large deformation increases the nucleation rate of recrystallization, which makes the grain size decreased, and the grain refinement degree is obvious, which is less than 15um.

\section{Acknowledgements}

The project is supported by following funds: the National Natural Science Foundation of China (Grant No.51475247), the Science and Technology Project of Zhejiang Province (2016C31018). 


\section{References}

[1] B. Yan. Study on microstructure of workpiece in multi-wedge cross wedge rolling[D]. University of Science and Technology Beijing,2010.

[2] B. Yan, X.D. Shu,Z.H. Hu. Development and status of study on microstructure evolvement regularity of workpiece of cross wedge rolling[J]. China Metallurgy,2008,18(2):8-10.

[3] Qiang Li,Michael Lovell. Cross wedge rolling failure mechanisms and industrial application[J]. The International Journal of Advanced Manufacturing Technology,2008,37(37):265-278.

[4] Fu Qiang Ying,Bo Song Pan. Analysis on temperature distribution in cross wedge rolling process with finite element method[J]. Journal of Materials Processing Tech,2007,187(4):392-296.

[5] Y.C. Lin, M.S. Chen, J. Zhong. Flow stress behaviors of 42CrMo steel during hot compression[J]. J. Cent. South Univ. (Science and Technology),2008,39(3):549-553.

[6] Y.C. Lin, M.S. Chen, J. Zhong. Effects of deformation degree on plastic formation and microstructure evolution of 42CrMo steel[J]. J. Cent. South Univ. (Science and Technology),2008,39(5):1005-1010.

[7] M.T. Wang, F.S. DU, X.T. Li, Y.Z. Zheng. Predication on austenite microstructure evolution during hot deformation of cross wedge rolled shaft[J]. Acta metallurgica sinica, 2005, 41(2): 118-122. 